\title{
Physics Potential of Super-K Gd
}

\author{
Charles Simpson* for the Super-Kamiokande Collaboration \\ University of Oxford \& University of Tokyo, Kavli IPMU \\ E-mail: charles.simpson@physics.ox.ac.uk
}

\begin{abstract}
The next stage of the highly successful Super-Kamiokande experiment is to load gadolinium (Gd) sulphate at $0.2 \%$ by mass. Gadolinium has a very large cross section for thermal neutron capture, which produces a cascade of gamma rays totalling $8 \mathrm{MeV}$. This is much easier to detect than the 2.2 MeV gamma ray from neutron capture on hydrogen that is currently used for neutron tagging. By tagging events which produce neutrons, background rates are radically reduced for some analyses. Super-K has published the best limits on the diffuse supernova neutrino background (DSNB, also called supernova relic neutrinos); neutron tagging with Gd will enable detection of the DSNB in 10 years from Gd loading. In the event of a core collapse supernova in our galaxy, neutron tagging with Gd will give new insight in to the dynamics of the neutrino burst, and a more accurate measurement of the direction to the supernova. Prior to a very close supernova $(<1 \mathrm{kpc})$, the late stages of stellar burning would be detected, giving even earlier supernova warning, and probing a never before observed stellar process. Super-K sets the best limits on proton decay - this will be improved by the addition of gadolinium as the majority of atmospheric neutrino induced backgrounds have one or more neutrons in the final state. Additionally hundreds or thousands of reactor neutrinos will be detected, and spallation induced backgrounds, which create dead-time for many analyses, will be reduced. The expected benefits of the addition of Gd are explored for these various physics analyses.
\end{abstract}

The 39th International Conference on High Energy Physics (ICHEP2018)

4-11 July, 2018

Seoul, Korea

${ }^{*}$ Speaker. 


\section{Introduction}

The Super-Kamiokande (SK) experiment consists of a $50 \mathrm{kt}$ tank of ultra-pure water instrumented with PMTs, and has been highly successful in neutrino detection and nucleon decay searches. The next stage of $\mathrm{SK}$ will be the addition of gadolinium sulfate $\left(\mathrm{Gd}_{2}\left(\mathrm{SO}_{4}\right)_{3}\right)$ to the detector water, and will be referred to as SK-Gd. Some details of implementation of the project are covered in a talk in the same session of ICHEP[1]. Detection of the diffuse supernova neutrino background (DSNB), also known as supernova relic neutrinos ( $\mathrm{SRN}$ ), is the main motivation for SK-Gd [2], but there will also be improvements to the detection of supernova burst neutrinos, and pre-supernova neutrinos from Si burning in the case of a very nearby core collapse supernova (CCSN), among other benefits [3].

SK currently detects neutrons through $2.2 \mathrm{MeV} \gamma$-ray produced by thermal neutron capture on H. The resulting $\gamma$-ray can scatter an electron, which emits Cherenkov light and be detected, but the amount of visible energy is low. Neutron capture candidates are searched for in coincidence with a higher energy event. This massively reduces backgrounds for neutron producing interaction channels such as inverse beta decay, as accidental coincidences between uncorrelated backgrounds are rare.

Naturally abundant isotopes of Gd have some of the highest thermal neutron capture cross sections, adding 0.2(0.02) \% Gd sulfate by mass to the SK ultra pure water will lead to 90(50) \% of captures occurring on Gd. Thermal neutron capture on Gd leads to a $\gamma$-ray cascade of around $8 \mathrm{MeV}$, leading to around 4-5 MeV of visible energy, which can be detected much more efficiently and with better position resolution. Furthermore, as the total thermal neutron capture cross section is increased, the time between the two parts of the event is decreased, making accidental coincidences even less likely.

\section{The Diffuse Supernova Neutrino Background}

The neutrinos emitted from all previous supernova ( $\mathrm{SN}$ ) in the universe form an isotropic flux, red-shifted to a low energy, which has never been detected. Measurement would provide information on the typical emission spectrum and rate of SN in the universe. All neutrino flavours are present, but electron anti-neutrinos are the best channel for detection at SK through the inverse beta decay (IBD) channel $\bar{v}_{e}(p, n) e^{+}$. Current limits published by the SK collaboration ( [4] for SK I-III, and [5] for SK IV with neutron tagging on hydrogen ) are background-limited, so will not benefit much from simply accumulating further live time. Using neutron tagging on $\mathrm{Gd}$, the expected background rate falls to 34 per 10 years, with expected signal of 11.9-31.2 events per 10 years, depending on the neutrino spectrum temperature, which means that positive detection of the DSNB will be in reach for SK-Gd [3].

\section{Benefits to Supernova Burst Detection}

In the event of a galactic or near-galactic SN, SK is able to discover the direction to the SN by reconstructing the direction of electrons scattered by the abundant neutrinos. Positrons from IBD in this case are background, as they do not indicate the direction of the incoming $\bar{v}_{e}$. By using 
neutron tagging on $\mathrm{Gd}$, it is possible to eliminate the majority of IBD events, thus improving the SN pointing ability of the detector. New studies [6] using the NK1 supernova model [7] have shown that angular resolution under $5^{\circ}$ can be achieved at over $14 \mathrm{kpc}$, improved from $10 \mathrm{kpc}$ without $\mathrm{Gd}$.

\section{Pre-supernova Neutrinos from Si Burning}

At the end of a massive star's life, $\mathrm{H}$ and $\mathrm{He}$ fusion rates are not sufficient to stabilise the star.

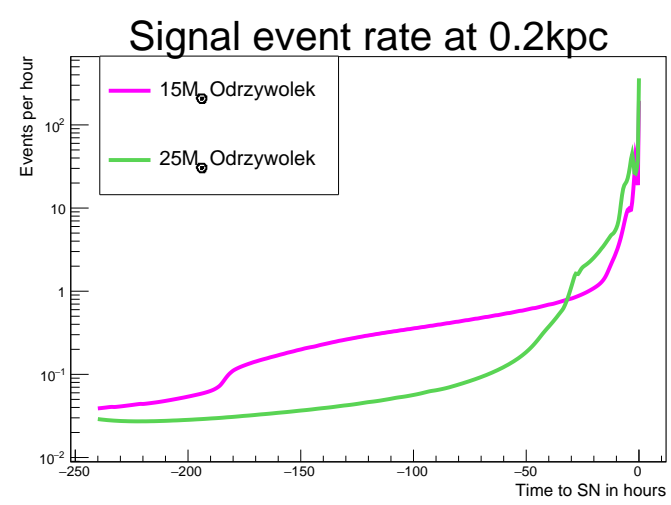

Figure 1: Event rate due to pre-supernova neutrinos in SK-Gd plotted against time before CCSN, based on the model of Odrzywolek et al. [8], before efficiencies are taken in to account, and assuming the star is 200 parsec away. The mean energy is also increasing during this time, so the change in the detected rate could be even more rapid due to detection thresholds. Contraction under gravity leads to increased temperatures and densities, heavier nuclei up to Fe can be fused, and neutrino emission becomes the largest source of cooling [8]. This process precedes a CCSN, and produces a rapidly evolving neutrino flux, as shown by Fig. 1. $\bar{v}_{e}$ will be more effectively identified in SK-Gd, even at low neutrino energies. So for a sufficiently close star a rapidly increasing flux of $\bar{v}_{e}$ could be detected and identified in SK-Gd prior to a CCSN. Detection would be challenging, as fluxes and energies are low: mean $\bar{v}_{e}$ energies are typically below the IBD threshold, so only the tail of the distribution would be detected. Backgrounds at SK are highest at low energy due to internal radioactivity, reactor neutrinos, and noise.

This pre-supernova signal could be detected hours or even days before the burst of neutrinos from core-collapse, which itself leads the optical signal by some hours. Therefore, detection of pre-SN neutrinos can be used to produce an additional early warning of a very nearby supernova. This will be used within the SK collaboration to prevent missing a supernova due to planned down-time, and could also provide an alert to the community.

In order to be detected in SK-Gd, $\bar{v}_{e}$ will need to exceed the IBD threshold of $1.8 \mathrm{MeV}$. For $e^{+}$to be detected they must produce enough Cherenkov radiation, and some photons are lost, so $e^{+}$below around $4 \mathrm{MeV}$ are not reconstructed with full efficiency. Neutron capture on Gd can be detected regardless of the energy of the $e^{+}$, so there is some detection efficiency right down to the reaction threshold, although without the coincidence with the positron the backgrounds are higher.

Estimates have been made of the efficiencies of detection for neutron captures on $\mathrm{Gd}$, and of low energy positrons in coincidence. These have been used to calculate the amount of extra SN early warning that SK-Gd could provide. The performance of SK-Gd will depend on the actual level of background, which is somewhat uncertain until in-situ measurements are made, and which depend on nuclear reactor activity in Japan as reactor $\bar{v}_{e}$ are a major background. Full details of this analysis will be published at a later date. 


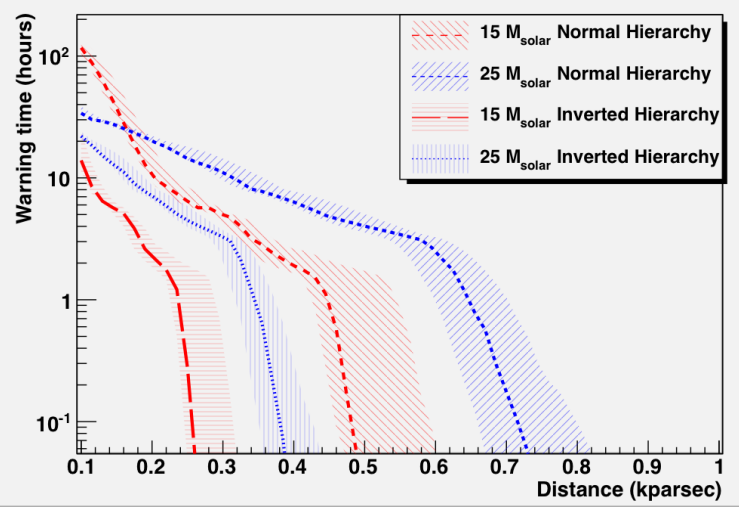

Figure 2: Time before the onset of CCSN at which SK-Gd could detect pre-SN neutrinos assuming a 1 per 2 year false alarm rate. This is dependent on unknown future background rates, mainly due to nuclear reactor activity, as well as the mass hierarchy, shown by the shaded bands.
Betelgeuse is given as an example of a nearby candidate for a future CCSN. Fig. 2 shows that SK-Gd would be able to give a warning of up to 60 hours (assuming $15 M_{\odot}$ and $150 \mathrm{pc}$ away, normal $v$ mass ordering) before Betelgeuse becomes a CCSN, assuming a 1 per 2 year type-II error rate (it is thought an expected type-II error rate of 1 false alarm per 2 years would be acceptable for internal use in the collaboration, but a lower rate could be required for any wider community alert). Maximum range for a $3 \sigma$ detection of this signal would be around 900 parsecs, which covers 41 red super giants (although some of these may not be massive enough for Si burning).

\section{Conclusion}

The Gd loaded phase of Super-Kamiokande will lead to the detection of the DSNB within a few years of full loading. Other benefits include increased ability to point to the source of SN burst neutrinos, and the ability to detect pre-SN neutrinos from Si burning for a very nearby star.

\section{References}

[1] SUPER-KAMIOKANDE collaboration, Ll. Marti-Magro, The Road to SuperK-Gd, PoS ICHEP2018 (2018) .

[2] J. F. Beacom and M. R. Vagins, GADZOOKS! Anti-neutrino spectroscopy with large water Cherenkov detectors, Phys. Rev. Lett. 93 (2004) 171101 [hep-ph/ 0309300$].$

[3] Super-KamiokAnde collaboration, H. Sekiya, The Super-Kamiokande Gadolinium Project, PoS ICHEP2016 (2016) 982.

[4] SUPER-KAmiokAnde collaboration, K. Bays et al., Supernova Relic Neutrino Search at Super-Kamiokande, Phys. Rev. D85 (2012) 052007 [1111. 5031].

[5] SuPer-Kamiokande collaboration, H. Zhang et al., Supernova Relic Neutrino Search with Neutron Tagging at Super-Kamiokande-IV, Astropart. Phys. 60 (2015) 41 [1311. 3738].

[6] T. Yano, Private communication, 2018.

[7] K. Nakazato et al., Supernova Neutrino Light Curves and Spectra for Various Progenitor Stars: From Core Collapse to Proto-neutron Star Cooling, Astrophys. J. Suppl. 205 (2013) 2 [1210 . 6841 ].

[8] A. Odrzywolek and A. Heger, Neutrino signatures of dying massive stars: From main sequence to the neutron star, Acta Physica Polonica B 41 (2010) . 